\title{
Alternative Design Concepts for Multi-Circuit HTS Link Systems
}

\author{
Amalia Ballarino
}

\begin{abstract}
Superconducting cables for power transmission usually contain two conductors for DC application, or three conductors for AC, with high voltage insulation. In contrast, for some applications related to accelerators it is convenient to transfer high currents via superconducting links feeding a number of circuits at relatively low voltage, of the order of a kilovolt, over distances of up to a few hundred meters. For power transmission applications based on cooling via sub-cooled liquid nitrogen, suitable HTS conductors are only available in the form of tape, and a multilayer variant can be envisaged for the multi-circuit links. However, where cooling to temperatures of the order of $20 \mathrm{~K}$ is feasible, $\mathrm{MgB}_{2}$ conductor, available in the form of both tape and wire, can also be envisaged and in the latter case used to assemble round cables. There are, therefore, two distinct topologies-based on the use of wires or tapes- that can be envisaged for use in applications to multi-circuit link systems. In this paper the merits of the two approaches are compared, and case studies related to applications to the LHC are presented.
\end{abstract}

Index Terms-BSCCO, HTS, $\mathrm{MgB}_{2}$, superconducting link, superconductors, YBCO.

\section{INTRODUCTION}

$\mathbf{T}$ HE use of superconducting links in accelerator systems enables the remote powering of cryo-magnets. The benefits derived from the adoption of this technology [1] are associated with an acquired flexibility in the location of the powering equipment. In particular, current leads and associated cryostats and control devices can be moved away from regions near the beam to locations, possibly near the power converters, more easily accessible and radiation-free. This implies: 1) additional free space in the beam's areas that becomes available for accelerator components 2) safer long-term operation of the powering equipment located in a radiation-free environment and 3) safer and easier access of personnel to power converters, current leads and related control equipment for maintenance, repair, diagnostic and routine test interventions-e.g. electrical insulation tests of magnet circuits during the accelerator shut-down periods. The latter implies also a reduced time of interventions, with consequent gain in machine availability. Besides the general application conceived for the powering of the accelerator magnets, a multi-conductor semi-flexible electrical transfer line would also serve other purposes. It is the case of links that could be used for the powering of special superconducting magnets,

Manuscript received August 02, 2010; accepted November 24, 2010. Date of publication January 06, 2011; date of current version May 27, 2011.

The author is with CERN, European Organization for Nuclear Research, 1211 Geneva 23, Switzerland (e-mail: amalia.ballarino@cern.ch).

Digital Object Identifier 10.1109/TASC.2010.2096378 installed locally in the accelerator, and links for equipment installed in the experimental areas.

HTS power transmission cables contain up to three conductors operated most frequently in $\mathrm{AC}$ mode and at maximum currents of the order of $4 \mathrm{kA}$. Cooling is provided by sub-cooled nitrogen - liquid hydrogen is an option proposed for $\mathrm{MgB}_{2}$ cables - and high voltage insulation of up to about $140 \mathrm{kV}$ is required. In contrast with such power transmission cables, superconducting links designed for the cold powering of accelerator magnet systems are required to transfer high-currents for the feeding of dozens of magnet circuits operated at relatively low voltages. Multi-circuit high-current links containing DC cables electrically insulated at voltages of the order of the kilovolt are therefore required. Cooling with helium at one of the several different temperature levels that can be made available by the cryogenic system is a viable option. The use of helium cryogenics offers the possibility of operating HTS with a generous temperature margin that can compensate for localized or distributed increased heat loads due to conductor movement, radiation losses or non-conformities of the link's cryogenic envelope, and thereby contribute to safe operating conditions.

Among the different HTS conductors, YBCO, BSCCO 2223 and $\mathrm{MgB}_{2}$ are available in the form of tape. $\mathrm{MgB}_{2}$ is also available in the form of wire. For multi-circuits cables one can therefore envisage various topologies, optimized to match the electrical, geometrical and mechanical properties of the conductor.

\section{SUPERCONDUCTING LINKS FOR THE LHC}

The powering of the LHC magnets is performed via power converters located in tunnel alcoves-with the exception of the dipole correctors whose converters are in the main tunnel. Warm cables connect the power converters to the leads integrated in cryostats in line with the beam. The use of superconducting links would bring in the advantages listed in Section I and also enable the removal of the heavy warm cables.

\section{A. Potential Applications to the LHC Machine}

A program for the development of $\mathrm{MgB}_{2}$ high-current cables and associated links was launched in the framework of the LHC Interaction Region Upgrade-Phase I [2], which was envisaging the remote powering of the low-beta triplets of the high luminosity experiments via current leads located, near the power converters, in underground areas at up to $100 \mathrm{~m}$ distance from the magnets [3]. A semi-horizontal—few meters of difference in height-superconducting link was proposed for connecting, in the tunnel, the current leads to the triplets. In this context, $\mathrm{MgB}_{2}$ cables of about $3 \mathrm{~m}$ length were developed and tested at $4.2 \mathrm{~K}$ at currents of up to 17800 A [4]. The LHC Upgrade-Phase I, now merged with Phase II, was recently re-scheduled to 2020, 
and the program re-optimized to serve for wider purposes [5]. It is now envisaged to remove the power converters and current leads to the surface. In this case, the powering of the new magnets would be performed via vertical links - up to about $100 \mathrm{~m}$ of difference in height-transferring the current from the surface to the accelerator underground areas. Horizontal links in the tunnel would then bring the current to the magnets.

While the new powering layout for the LHC upgrade is still under study, a program has been launched to investigate the possibility of using superconducting links in the context of the R2E (Radiation to Electronics) consolidation program. In view of the potential radiation-induced failures to electronics [6] - mainly because of single particle event effects-there is an interest in removing the LHC power converters to radiation free areas and in using superconducting links for the electrical connection to the magnets. This is the case at Point 7 (P7), where a semi-horizontal link would transfer the current from a safe underground area to the tunnel, and at Point 1 (P1) and Point 5 (P5), where the power converters would be re-located at the surface and vertical links of the type in study for the LHC upgrade would be needed. The total length of these links would be of the order of $600 \mathrm{~m}$. At P1 and P5 the precise length will depend on the final location of the surface buildings housing the power converters and the current leads. Six links, two at each of the three interaction points, are needed. The modification of the powering layout for the R2E consolidation program should preferably be ready earlier than the installation for the new upgrade, i.e. as from 2016.

The R2E consolidation activity concerns all the LHC electrical circuits presently fed via current leads located in the tunnel at P7, P1 and P5, with the exception of those feeding the Inner Triplets, whose powering is studied within the upgrade program. Those leads and associated cryostats will also later be removed and located near the power converters at the surface.

The links at P1 contain 48 cables rated at $600 \mathrm{~A}$. The links at $\mathrm{P} 5$ and $\mathrm{P} 7$ contain 12 cables rated at $120 \mathrm{~A}, 28$ cables rated at $600 \mathrm{~A}$ and 23 cables rated at $6000 \mathrm{~A}$. The total required current capability is respectively $29 \mathrm{kA}$ and $156 \mathrm{kA}$. The cables operate in quasi-DC mode (slow ramping).

\section{B. Cooling}

The temperature levels that can be made available by the LHC cryogenics [7] are: supercritical helium at about $5.5 \mathrm{~K}$ and 3.5 bar, helium gas at about $20 \mathrm{~K}$ and $1.3 \mathrm{bar}$ and helium gas in the temperature range from $50 \mathrm{~K}$ to $75 \mathrm{~K}$ and $18.5 \mathrm{bar}$. The latter option could possibly be adopted for the cooling of cables made with YBCO or BSCCO conductors. As a baseline option, supercritical helium at $5.5 \mathrm{~K}$ is used for the cooling of the cables. The cryogenic envelope is a flexible Cryoflex line incorporating a thermal shield cooled by helium gas entering at about $20 \mathrm{~K}$. The external diameter is $220 \mathrm{~mm}$ and the diameter of the corrugated pipe containing the superconducting cables is $84 / 92 \mathrm{~mm}$.

\section{Superconducting Materials}

The superconductors studied for application to superconducting links are: pre-reacted $\mathrm{MgB}_{2}$ wire or tape, YBCO and BSCCO 2223 tapes. For the $\mathrm{MgB}_{2}$, the maximum operating temperature is specified to be $25 \mathrm{~K}$ in the field generated by the cable. For the purpose of this study, the YBCO and BSCCO
TABLE I

CONDUCTORS FOR SUPERCONDUCTING LINKS

\begin{tabular}{lcccccc}
\hline \hline & & $\begin{array}{c}\Phi \\
(\mathrm{mm})\end{array}$ & $\begin{array}{c}\mathrm{W} \\
(\mathrm{mm})\end{array}$ & $\begin{array}{c}\text { Th } \\
(\mathrm{mm})\end{array}$ & $\begin{array}{c}\text { Tmax } \\
(\mathrm{K})\end{array}$ & $\begin{array}{c}\mathrm{Ic}^{\mathrm{a}} \\
(\mathrm{A})\end{array}$ \\
\hline $\mathrm{MgB}_{2}$ & wire & 1.1 & - & - & 25 & $>400$ \\
$\mathrm{MgB}_{2}$ & tape & - & 3.7 & 0.67 & 25 & $>400$ \\
$\mathrm{YBCO}$ & tape & - & 4 & 0.1 & 35 & $>400$ \\
$\mathrm{BSCCO} 2223$ & tape & - & 4 & 0.2 & 35 & $>400$ \\
\hline \hline
\end{tabular}

${ }^{a}$ The magnetic field to which the conductors are exposed is $<0.5 \mathrm{~T}$.

tapes are allowed to operate at up to $35 \mathrm{~K}$. Dimensions (diameter, $\Phi$, width, $W$, and thickness, $T h$ ) and electrical properties (critical current, $I c$ at maximum operating temperature, $T \max$ ) of the conductors studied for this application are summarized in Table I [8]-[12]. The $\mathrm{MgB}_{2}$ wire incorporates about $14 \%$ of $\mathrm{Cu}$ stabilizer [9]; the YBCO tape has a total copper layer thickness of $40 \mu \mathrm{m}$ [11], [12].

\section{Superconducting Cables}

The cables are assembled, electrically insulated and prepared for pulling into the cryogenic envelope. The full multi-circuits cable assembly is housed within a corrugated pipe of $84 / 92 \mathrm{~mm}$ diameter that contains the flowing helium gas. Each cable is fully transposed and consists of twisted superconducting strands and additional copper stabilizer. The total cross section of stabilizer depends on the protection requirements accordingly to the time constants of the respective LHC electrical circuits $(<5 \mathrm{~s})$. The twist pitch is chosen to accommodate the mechanical properties of the conductors. Minimum bending radii, corresponding to zero $I c$ degradation, of $80 \mathrm{~mm}, 30 \mathrm{~mm}$ and $7 \mathrm{~mm}$ have been used respectively for the $\mathrm{MgB}_{2}$ reacted wires and tapes, for the BSCCO 2223 and for the YBCO tapes. The insulation of each cable is assured by a multi-layer wrapping of overlapped Kapton polyimide tape. The total thickness of the insulation between conductors is from $0.25 \mathrm{~mm}$ to $0.5 \mathrm{~mm}$ depending of the type of cable. The $6 \mathrm{kA}$ cables are designed to withstand an electrical insulation voltage of $1.5 \mathrm{kV}$ when tested in helium gas environment at room temperature and at a pressure of $1.5 \mathrm{bar}$. The electrical insulation voltage of the $120 \mathrm{~A}$ and $600 \mathrm{~A}$ cables is $1 \mathrm{kV}$.

\section{Superconducting Cables: Case Studies}

Several geometries of the two types of multi-circuit cables needed for the R2E consolidation program have been studied. In the following sections, the assembly of 12 cables for $120 \mathrm{~A}$, 28 cables for $600 \mathrm{~A}$ and 23 cables for $6 \mathrm{kA}$ will be referred to as Type 1, while the assembly of 48 cables for $600 \mathrm{~A}$ will be referred to as Type 2. Additional spare cables may be included in the assembly.

Three $6 \mathrm{kA}$ cables are assembled concentrically in layers of conductors. They feed the LHC circuits that contain two magnets coupled together by a shared output cable: the magnets are independently powered via two power converters and three current leads connected to a superconducting cable. Each cable shall be capable of transporting up to $6 \mathrm{kA}$. When only one of the magnets is powered, two cables carry up to $6000 \mathrm{~A}$ while the third cable is idle. When both circuits are powered, the sum 


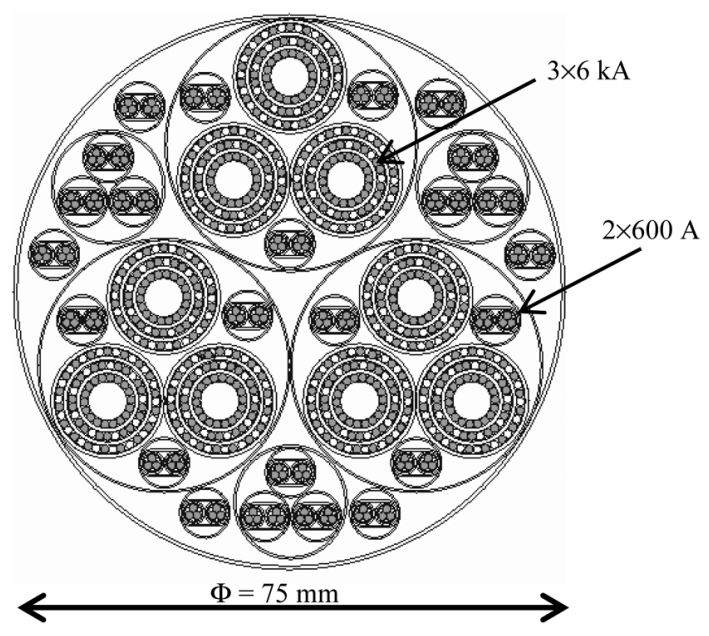

Fig. 1. $\mathrm{MgB}_{2}$ full multi-cable assembly containing 27 cables $6000 \mathrm{~A}$ and 48 cables rated at $600 \mathrm{~A}$ - total current capacity $190 \mathrm{kA}$.

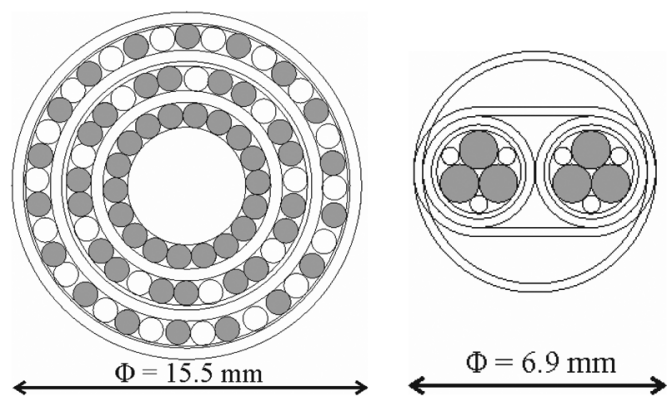

Fig. 2. Concentric 3-cable $6000 \mathrm{~A}$ assembly (left side) and twisted pair of $120 \mathrm{~A} / 600 \mathrm{~A}$ cables (right side). Grey circles are $\mathrm{MgB}_{2}$ wires, white circles are $\mathrm{Cu}$ wires.

of the currents in the three cables is zero. In this case, for constraints related to the control of the power converters, the maximum current transported by the shared cable is $3 \mathrm{kA} \mathrm{[13].}$

The $120 \mathrm{~A}$ and $600 \mathrm{~A}$ cables consist of twisted pairs powering the same electrical circuit. The difference in size is small, and for standardization they have been chosen to be identical.

\section{A. $M g B_{2}$ Wires}

The Type 1 cable assembly made with $\mathrm{MgB}_{2}$ wires (see Fig. 1) consists of:

1) nine $6 \mathrm{kA}$ concentric 3 -cable assemblies, i.e. 27 cables, arranged in groups of three in a triangular shape;

2) twenty-four $600 \mathrm{~A}$ twisted pairs, i.e. 48 cables.

The total current capacity is $190 \mathrm{kA}$ and the external diameter of the insulated multi-cable assembly is about $75 \mathrm{~mm}$.

Each $6 \mathrm{kA}$ cable consists of $18 \mathrm{MgB}_{2}$ wires. In the inner layer of the 3-cable assembly (see Fig. 2) the superconducting wires are wound on a bundle of copper strands, while in the central and outer layers stabilizing copper wires alternate with the $\mathrm{MgB}_{2}$ wires. The wires of each layer are helically wound with a constant and alternating twist pitch of $500 \mathrm{~mm}$.

The 600 A cable consists of three $\mathrm{MgB}_{2}$ wires twisted with three copper strands. Cables of opposite polarity are bound together to form a twisted pair of equivalent diameter $6.9 \mathrm{~mm}$ (see Fig. 2).

In the configuration shown in Fig. 1, when all the cables are powered at maximum current, the $6 \mathrm{kA}$ cables have a tangential

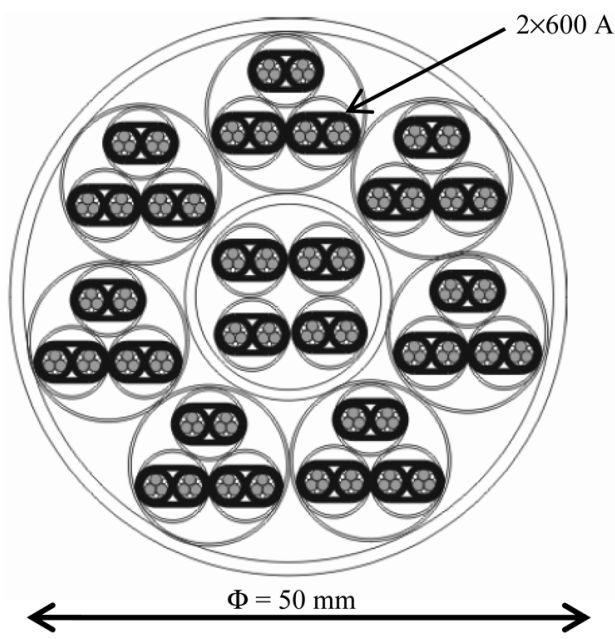

Fig. 3. $\mathrm{MgB}_{2}$ full multi-cable assembly containing 50 cables $600 \mathrm{~A}$-total current capacity $30 \mathrm{kA}$.

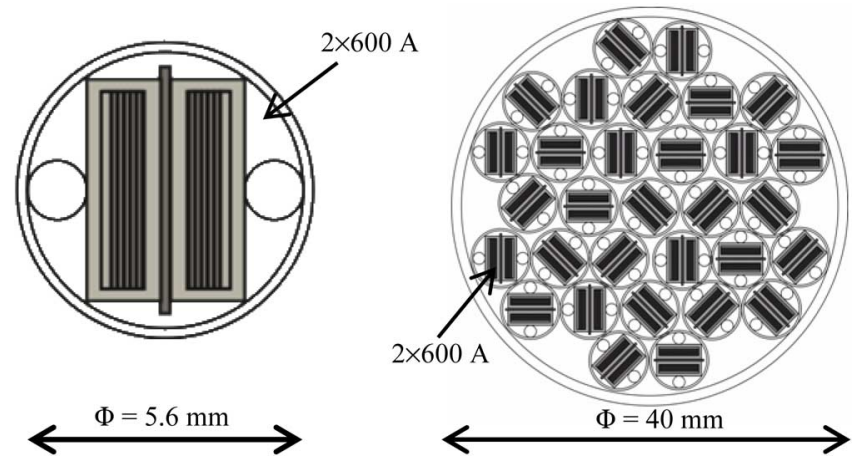

Fig. 4. YBCO twisted pair $600 \mathrm{~A}$ cable (left side) and YBCO full multi-cable assembly containing 54 cables $600 \mathrm{~A}$-total capacity $32 \mathrm{kA}$.

field component of few $\mathrm{mT}$ inside the inner layer and $\leq 30 \mathrm{mT}$ outside the outer layer, and a peak field of $0.28 \mathrm{~T}$ in the most inner part of the outer layer. In the same configuration, the 600 A cables have a peak field of $0.17 \mathrm{~T}$ in the contact area between the twisted pairs [14].

The Type 2 cable assembly (see Fig. 3) consists of twenty-five twisted pairs $600 \mathrm{~A}$ cables of the type shown in Fig. 2. The outer diameter of this multi-cable assembly is $50 \mathrm{~mm}$ and the total current capacity is $30 \mathrm{kA}$. The peak field, which occurs when all the cables are powered at maximum current, is $0.17 \mathrm{~T}$ [14].

\section{B. YBCO, BSCCO, and $M g B_{2}$ Tapes}

$\mathrm{YBCO}, \mathrm{BSCCO}$ and $\mathrm{MgB}_{2}$ tapes have similar electrical properties at the temperatures and low fields of interest for this application but significantly different mechanical properties. Also, the tape geometry does not facilitate a multi-cable design that shall accommodate some flexibility of the final assembly-in any direction-as well as compactness.

The low current cables are the most difficult to be arranged in a compact way. The $600 \mathrm{~A}$ cables are proposed to be made from three superconducting tapes sandwiched with copper strips in the case of $\mathrm{YBCO}$ and $\mathrm{MgB}_{2}$ conductors. The BSCCO cable does not need additional copper-the stabilizer is its silver alloy matrix. Pairs of insulated cables are then twisted together to form an envelope with an outer diameter of about $5.6 \mathrm{~mm}$ for the YBCO (see Fig. 4), $6 \mathrm{~mm}$ for the BSCCO and $7.5 \mathrm{~mm}$ for the 


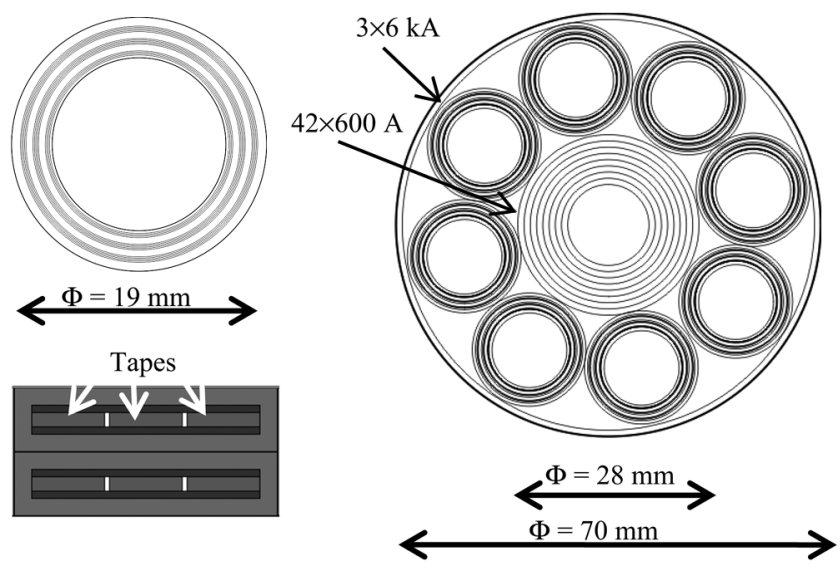

Fig. 5. YBCO 3-cable assembly (top left side), YBCO twisted-pair 600 A cable (bottom left side), Type 1 YBCO full multi-cable assembly (right side).

$\mathrm{MgB}_{2}$ - to be compared with the $6.9 \mathrm{~mm}$ of the $\mathrm{MgB}_{2}$ cable in Fig. 2. The Type 2 cable is then made from assembly of twisted pairs. The outer diameter of the multi-cable assembly containing 27 pairs of 600 A cables (see Fig. 4) is about $40 \mathrm{~mm}$ for BSCCO and $\mathrm{YBCO}$, and $50 \mathrm{~mm}$ for $\mathrm{MgB}_{2}$ - to be compared with $50 \mathrm{~mm}$ for the $\mathrm{MgB}_{2}$ multi-cable in Fig. 3 .

The $6 \mathrm{kA}$ 3-pole cable assembly is made from concentric layers of tapes and stabilizer helically wound around a former. The outer diameter is $19 \mathrm{~mm}$ (see Fig. 5). Each cable pole consists of two layers of nine tapes interleaved with copper strips. Cable poles are separated by $0.5 \mathrm{~mm}$ of insulation that includes $0.1 \mathrm{~mm}$ thick of polyimide film. The Type 1 cable contains eight such $6 \mathrm{kA}$ 3-pole cables arranged around a sub-assembly of twenty-one 2-pole $600 \mathrm{~A}$ cables (see Fig. 5). This sub-assembly, of outer diameter $28 \mathrm{~mm}$, consists of seven layers, each made up of three pairs of 3-tape cables, helically-wound around a $13 \mathrm{~mm}$ diameter flexible mandrel. The tapes are preassembled between copper foils as sketched in Fig. 5.

The 600 A multi-cable included in the Type 1 cable shown in Fig. 5 can also be used for the assembly of Type 2 cables - as an alternative to the geometry shown in Fig. 4. In this case, the number of cables rated for $600 \mathrm{~A}$ has to be increased from 42 to 48 , and one additional layer of three cable pairs is needed. The outer diameter of such an assembly made with YBCO tapes is $<30 \mathrm{~mm}$. The configuration shown in Fig. 5 could also be used with BSCCO. Use of $\mathrm{MgB}_{2}$ tape would inevitably incur increases in stiffness and outer diameter.

\section{CONCLUSION}

Several geometries of cables optimized for application to the LHC machine were studied. It is seen that multi-cable assem- blies with a total DC current capacity of up to $190 \mathrm{kA}$ can be made with $\mathrm{MgB}_{2}$, BSCCO and YBCO conductors, and their external diameter can in all cases be contained to values $\leq 75 \mathrm{~mm}$. For the types of applications being studied for the LHC, long lengths of conductor with qualified and homogeneous properties will be needed. YBCO is superior for mechanical properties; BSCCO has acceptable mechanical properties, is available in long lengths and incorporates within the tape sufficient stabilizer for protection; $\mathrm{MgB}_{2}$ is available in long lengths and its low cost makes it attractive.

In the next months short prototype multi-cables will be assembled and tested in nominal operating conditions. An installation is also being prepared at CERN for the test, planned for end 2011, of semi-horizontal multi-cable links Type 1 and Type 2 of up to $30 \mathrm{~m}$. This development will indicate the most appropriate choice of conductor and cable geometry.

\section{REFERENCES}

[1] A. Ballarino, K. H. Meß, and T. Taylor, "Extending the use of HTS to feeders in superconducting magnet systems," IEEE Trans. Appl. Supercond., vol. 18, pp. 1455-1458, June 2008.

[2] R. Ostojic, "Conceptual Design of the LHC Interaction Region Upgrade-Phase I," LHC Project Report 1163, Nov. 2008.

[3] A. Ballarino, "Design of an $\mathrm{MgB}_{2}$ feeder system to connect groups of superconducting magnets to remote power converters," J. Phys.: Conf. Ser., vol. 234, p. 012001, 2010.

[4] A. Ballarino, P. Chambouvet, P. Denis, A. Gharib, T. Miani, and G. Willering, "Results of first tests of $\mathrm{MgB}_{2}$ cables," TE-MSC Internal Note, to be published.

[5] L. Rossi, "Task force on LHC luminosity upgrade," CERN Document Server EDMS No. 1067046, March 2010.

[6] R. Losito, "Where are we with the long-term plans and the CERN wide radiation policy," in Proceeding of Chamonix Workshop, 2010, CERNATS-2010-026.

[7] S. Claudet, P. Gayet, and U. Wagner, "Specification of Four New Large 4.5 K Helium Refrigerators for the LHC," LHC Project Report 331, Dec. 1999.

[8] Columbus Superconductors, $\mathrm{MgB}_{2}$ Products [Online]. Available: http://www.columbussuperconductors.com/mgb2.htm

[9] S. Berta, A. Ballarino, S. Brisigotti, G. Grasso, A. Tumino, and D. Pietranera, "Development of $\mathrm{MgB}_{2}$ Conductors for application to superconducting bus," CERN Document Server EDMS No. 965302, Sept. 2008.

[10] Bruker. [Online]. Available: http://www.bruker-est.com/bscco-tapes html

[11] Bruker. [Online]. Available: http://www.bruker-est.com/ybco-tapes html

[12] SuperPower. [Online]. Available: http://www.superpower-inc.com/ content/products

[13] Y. Thurel, "LHC4-6-8kA-08V MQM-MQY Guide," CERN Document Server EDMS No. 1027850

[14] A. Ballarino et al., "Magnetic Field and Current Distribution in HTS Cables for Application to LHC," TE-MSC Internal Note, to be published. 